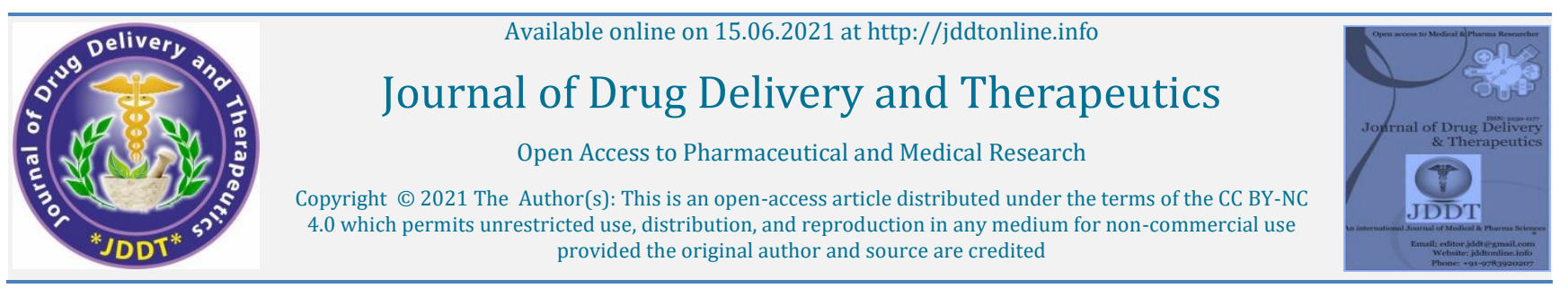

Open Access Full Text Article

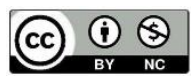

Review Article

\title{
In-Situ Forming Polymeric Drug Delivery Systems for Ophthalmic Use: An Overview
}

\author{
Pranal Chhetri *, Prithviraj Chakraborty, Debasmita Das, Tamanna Afnan \\ Department of Pharmaceutics, Himalayan Pharmacy Institute, Majhitar 737136, East Sikkim, India
}

\begin{tabular}{ll}
\hline Article Info: & \\
\hline$\square$ & Article History: \\
& Received 18 April 2021 \\
& Accepted 28 May 2021 \\
& Available online 15 June 2021
\end{tabular}

Cite this article as:

Chhetri P, Chakraborty P, Das D, Afnan T, In-Situ Forming Polymeric Drug Delivery Systems for Ophthalmic Use: An Overview, Journal of Drug Delivery and Therapeutics. 2021; 11(3-S):98-103 DOI: http://dx.doi.org/10.22270/jddt.v11i3-S.4874

\section{*Address for Correspondence:}

Pranal Chhetri, Department of Pharmaceutics, Himalayan Pharmacy Institute, P O Box 737136 Majhitar, East Sikkim, India

\section{Abstract}

Delivery of drug into the ocular region is hindered by the protective layers that encapsulate the eyes, it has always been a major problem to get an effective bioavailability of the active drug in the ocular region due to the low precorneal resident time of most of the ocular delivery systems specifically convention once such as ointment, solution and suspension, as a result, most of the delivery systems are not capable of effectively treating ocular diseases. Several works have and are being carried out to overcome this problem one of which is using in-situ forming polymeric systems. Ocular In-situ gelling systems are a novel class of ocular drug delivery systems that are initially in a solution form but instantaneously gets converted into a viscous gel upon introduction or installation in the ocular cavity from which the active drugs get released in a sustained manner. This sol-togel phase transition depends upon various factors like change in $\mathrm{pH}$, ion presence and change in temperature. Gel formed after the transformation has preferred viscosity along with bio-adhesive property, which increases the gel's resident time in the ocular area and also releases the drug in a prolonged and sustained manner unlike conventional eye drops and ointments. This review emphasizes various ocular in-situ systems namely, $\mathrm{pH}$ triggered, Ion activated, and Temperature triggered systems which have prolonged residence time in the cul-de-sac area of the eye, hence increasing the ocular bioavailability.

Keywords: In-situ gel, Ocular Drug delivery, Ocular Bioavailability, Polymer

\section{INTRODUCTION}

Drug delivery into the ocular region by using conventional delivery systems like drops and ointment requires frequent dosing the reason being that when they are introduced in the precorneal region they are quickly washed away due to the lachrymal nasal drainage and tear flow. Further, the efficacy of the administered drug is hindered by the barrier system of the eye making it nearly impossible for drugs to easily penetrate the eye ${ }^{1}$.A very small fraction of drug gets access to the active site i.e., approximately less than $1 \%$ of total drug instilled, this is why ocular delivery of the drug is a major challenging endeavour faced by the pharmaceutical scientist today ${ }^{2}$.

Over the last decade's number of novel works has been carried out to develop a newer and novel ocular delivery system to provide effective and easy to use systems by patients, in addition, to reduce the side effects and increasing the bioavailability as well, which could overcome the problems related to conventional once. One of the discoveries is a breakthrough which is in-situ gelling systems having significantly unique characteristics. A highly impressive number of these novel systems such as $\mathrm{pH}$ triggered ocular in-situ systems, ion activated systems and temperature triggered systems have been described in the literature which can be used as a perfectly efficacious alternative to conventional systems ${ }^{3}$.
Ocular in-situ gelling systems are delivery systems that are designed in such a way that they instantaneously change into gel form when dropping conventionally as a solution or suspension in the eye cavity. As these gels are viscous and have some bioadhesive property the residence time of the systems is likely higher than the conventional systems like eye drops, ultimately resulting in maximum ocular bioavailability 3,4 . The increase in the precorneal residence time leads to increased bioavailability consequently, increasing patient compliance. These in-situ gelling systems are highly acceptable and preferably convenient to be used by the patients and the features this system holds i.e., residence time up to several hours make it most impressive and convenient ${ }^{4}$. These attractive features of this novel ocular systems are due to the use of polymers that have an ability to transform from a sol-to-gel form in the presence or due to the change in various physiochemical environment as disused already, that are; $\mathrm{pH}$, ions and temperature of the cul-de-sac area of the eye $\mathrm{e}^{4,5}$

Some examples of the polymers used in the different in-situ systems are as follows; $\mathrm{pH}$ triggered system using Carbopol, ion activated systems using alginates such as sodium alginate and temperature triggered systems using xyloglucan ${ }^{5}$. Ideally, an ocular in-situ forming polymeric systems in solution form must have minimum viscosity and must be free-flowing for easy installation but after installation when it gets converted to a gel form it must have a slightly higher viscosity and must not be free-flowing and must be capable 
of resisting the shear force that it will experience in the culde-sac area of the eye ${ }^{6}$.

\section{ANATOMY OF THE EYE AND OCULAR DRUG PENETRATION}

The human eye is broadly divided into two distinct segments namely, the anterior and the posterior segments. The anterior segment consists of the conjunctiva, pupil, anterior chamber, lens, cornea iris, culinary body, aqueous humor, and trabecular meshwork. The posterior segment consists of the sclera, choroid, optical nerve, virtuous humor, retina, and macula. The cornea forms the outermost layer of the eye. It is found directly on the front side of the iris and pupil; it is an avascular and transparent part of the eye. It is further divided into five layers ${ }^{7}$. The corneal epithelium, which is the outermost layer of the cornea, Bowman's layer which is very thin, the corneal stroma, Descemet's membrane and the last innermost layer i.e., the corneal endothelium. The permeability of the drugs by the cornea is the most important factor that decides the aqueous humor drug concentrating. For the hydrophilic drugs, it is difficult for them to penetrate due to the epithelium layer thus epithelium acts as a rate-limiting layer or barrier for hydrophilic drugs. And for a lipophilic drug the rate-limiting layer is the corneal stroma due to the presence of hydrophilic collagen which is highly organized 8 .

The Conjunctiva, a thin and clear membrane, covers parts of the front surface of the eye and the inner surface of the eyelids. It consists of goblet cells and non-keratinized epithelium cells. Its main function is to protect the eyes as it has goblet cells it secrets mucus, preventing the entry of microbes in the eye and lubricates the eye ${ }^{8,9}$. Conjunctiva in the human eyes covers 17-times the surface that is covered by the cornea, and drugs permeate more from the conjunctiva than from the cornea facilitating higher drug absorption from the conjunctiva. But drug absorbed from the conjunctiva is not that significant as a large number of lymphatics and blood capillaries are present in the conjunctiva resulting in the loss of a considerable amount of drug into the blood and ultimately resulting in the low ocular bioavailability 9 .

Now talking about the aqueous humor it simply includes a clear watery fluid inside the front of the eye. It is nonvascular and transparent which allow the light to get through it. Aqueous humor works to provide nutrients to the eye and maintains the eye's normal shape and pressure. It has an excessive amount of ascorbate about 15 -fold the amount present in plasma, the $\mathrm{pH}$ of the aqueous humor is usually $7.2^{10}$.

The eye consists of a white portion known as sclera, it has good elastic property, is opaque and full of collagen fibres. The drug which is hydrophilic in nature generally penetrate through the sclera without much hindrance as compared to cornea and conjunctiva, this is due to the presence of proteoglycans in between the collagen mess which is aqueous in nature and through which the hydrophilic substance can easily diffuse through which is a wisher alternative than crossing via cell membrane ${ }^{10,11}$. The functions of the sclera include its serves as a protective layer of the eye and maintain intraocular pressure. The sclera receives or has a very low blood supply, some of the blood vessels run across the sclera but sclera itself is considered avascular. The retina where the process of vision formation begins when the light is converted into signals by the specialized cell known as photoreceptor cells present in the retina which are further interpreted inside the brain to create a vision. Due to its thick layer of cells substances with higher molecular weight finds it difficult to get through ${ }^{11}$.

\section{CHALLENGES IN DEVELOPING AN OPHTHALMIC DRUG DELIVERY SYSTEM}

\section{Anatomy and physiology of the eye}

A lot of impressive reviews are present in the books of literature from where we can get a clear view on anatomy and physiology of the eye with relation to ocular drug delivery. The anatomical and physiological features of the eye considerably interfere with the absorption and transport of administered active drug. Firstly, the primary features of the eye that is tear secretion, blinking of the eye and nasolacrimal drainage along with reflex action of the eye affects the precorneal residence time of the delivery system containing the active drug 12 .

Tears are almost every time secreted in the eye cavity for moistening the outer surface of the eye. These tears generally wash away the drug substance permanently after administration in a very short period, along with that it has an anti-infectious property due to the presence of immunoglobulins and the lysozyme, causing increased tear secretion after administration as a protective response. Then comes another problem that is nasolacrimal drainage, which is a process of drainage of lachrymal fluids through the nasolacrimal pathway that reaches pharynx and eventually oesophagus ${ }^{13}$.

\section{Delivery of drug to the internal regions of the eye}

Ocular Penetration of drugs administered systemically: Various barriers are present in the eye to prevent the transport of various unwanted substances into the eye, one of which is the blood-eye barrier. Aqueous humor;a clear aqueous liquid that is needed to maintain the intraocular pressure is formed through the ciliary process by ciliary epithelium. The ciliary epithelium is considered as an ultrafilter due to its preventive nature as it guards the passage or transport of substance with higher molecular weight including antibiotics and proteins. During the formation of aqueous humor some substances can be secreted in it, preferably those molecules which are having low molecular weight. Another barrier i.e. the blood-aqueous humor barrier gets disrupted when some inflammation occurs in the eye due to some ocular disease or infection. At this disrupted stage some of the drugs can get access to the aqueous humor and eventually reaches the anterior parts of the eye. Another highly viscous barrier present in the eye is the bloodvitreous humor barrier. Due to its high viscosity, it is extremely difficult for a drug molecule to diffuse through it. Drug delivery to the posterior part of the eye is considered highly difficult and an extremely challenging endeavour ${ }^{13,14}$.

Ocular penetration of locally administered drugs: Active Drug substance which is intended to work or show its effects inside the eye and not in the outer surface has to pass through the protective barriers and layers of the eye to enter inside. A general agreement exists that says, the trans corneal route is the most essential one for the transport of drugs into the eye, however, another route i.e. non-corneal is also having some significant importance in contributing to the absorption of some drug in the ocular region, for example, timolol. Additionally, many blocking agents used in ocular diseases shows high permeability to the sclera. A tear that is secreted has a primary function to keep the cornea clear, healthy, and moist which is evenly spread with the help of eyelids as a result of blinking, this blinking can change or modify the pattern and rate of trans corneal absorption ${ }^{15}$. 


\section{OCULAR IN-SITU GELLING SYSTEMS}

Ocular In-situ gelling system is ocular drug delivery systems which can undergo phase transition upon some physiochemical change from a solution or suspension to a gel form which is having some viscoelastic and sometimes bioadhesive property. The so formed gel results in the increased bioavailability along with it shows a prolonged and a sustained drug release pattern ${ }^{16}$.

Pharmaceutical industries with the help of their formulation development departments have made a significant amount of progress in this polymer technology which has an impressive feature of transforming instantaneously to a viscous gel from a free-flowing clear liquid. As already been discussed before; these formulations before installation are free-flowing liquid but undergo phase transition after installation in the ocular region in a very short period. Three methods namely, $\mathrm{pH}$ triggered, ion activated, and temperature triggered have been till now used to formulate an ocular in-situ gelling system ${ }^{17}$.

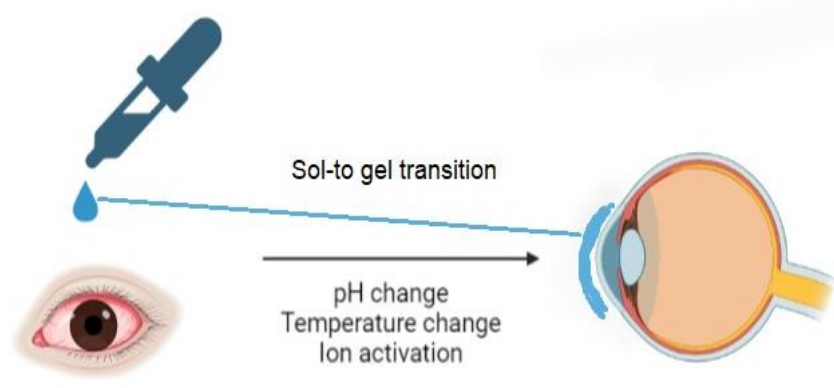

Figure 1: Mechanism of in-situ gel formation in the ocular region.

\section{Ion activated systems}

Ion activated systems as the name itself describe that phase transition is activated by some ion presence. The freeflowing liquid is converted to a viscous gel after installing it to the ocular region due to the change in ionic strength. For example, sodium alginate forms a viscoelastic gel which is clear in the presence of divalent cations such as calcium which is found in the tear fluid ${ }^{18}$.

\section{Polymers used in ion activated systems:}

Alginate/ Alginic acid: Alginic acid also known as algin or alginate is an anionic polysaccharide that is found abundantly in the cell walls of brown algae which is a sea weed and is also found in some types of bacteria. It is hydrophilic in nature and forms a viscous gel when it comes in contact with water. Structurally it is a copolymer having a linear shape with homopolymeric blocks of $(1 \rightarrow 4)$-linked $\beta$ D-mannuronate (M) and $\alpha$-L-guluronate $(G)$ residues, respectively, covalently linked together in different blocks or sequences. The most widely used alginate in in-situ gel technology is the sodium salt of alginic acid i.e., sodium alginate. Its phase transition from sol-to-gel is due to its interaction with the divalent action i.e., calcium ion $(\mathrm{Ca} 2+)$ found in the tear fluid ${ }^{19}$.

Pectin: Pectins belongs to the family of heteropolysaccharides, which is found abundantly in the primary and middle lamella and cell walls of terrestrial plants. Pectin is produced commercially from citrus fruits as is usually used as a gelling agent in the food industry. Structurally they are rich in $\alpha$ - $(1,4)$-d-galacturonic acid, various other distinct polysaccharide is identified within the pectin. It is soluble in water which is the most advantageous feature of the pectin that allows the avoidance of other solvents like organic solvents to be used in the delivery system $^{19,20}$.

Gellan gum: Gellan gum is another water-soluble anionic polysaccharide that is employed to produce ion-activated hydrogels. It is a having a linear heteropolysaccharide having a repeating unit of tetrasaccharide, which consist of Dglucose and one of each residue of L-rhamnose and Dglucuronic acid. Gellan gum is categorized into two groups; low acyl and high acyl depending upon the number of acetate groups attached to the polymer. Electrolytes like $\mathrm{Ca} 2^{+}, \mathrm{Na}^{+}$ and $\mathrm{Mg}^{+}$present in the lachrymal fluid is responsible for inducing gel formation upon administration of the formulation as a free-flowing liquid solution into the precorneal area ${ }^{20}$.

\section{2. pH triggered systems}

$\mathrm{pH}$ triggered systems can be defines as those oculars in-situ gel-forming systems that upon installation conventionally as an eye drop within a short period gets converted to a gel due to its exposure to different $\mathrm{pH}$ level (here it is $\mathrm{pH}$ of precorneal area).This happens due to the presence of $\mathrm{pH}$ sensitive substances or polymers in the formulation. At low $\mathrm{pH}(\mathrm{pH} 4-4.4)$ the prepared formulation remains as a freeflowing solution but upon instillation, it gets converted to a viscous gel due to a higher $\mathrm{pH}$ of lachrymal fluid that is $\mathrm{pH}$ $7.4^{21}$.

\section{Polymers used in pH triggered systems:}

Carbopol (Polyacrylic acid): Carbopol is a synthetic high molecular weight polymer of acrylic acid also known as polyacrylic acid. Carbopol may be cross linked with an allyl ether of pentaerythritol or allyl ether of propylene or maybe homopolymers of acrylic acid. The groups present mainly carboxylic group in the carbopol, releases or accept protons at a high and low value of $\mathrm{pH}$, respectively. It shows gel phase transition from sol to gel when the $\mathrm{pH}$ is raised above $5.5^{22}$.

\section{Temperature triggered systems}

Temperature triggered systems can be defined as those oculars in-situ gel-forming systems that have the ability to transform from a free-flowing liquid to a viscous gel, due to the temperature of the ocular region. The phase transition of the formulation to a gel from a solution is basically due to the temperature-sensitive polymers incorporated in it. Temperature triggered in-situ systems are the most widely studied topic among other ocular in-situ gel-forming systems. At lower temperature, this formulation is in the form of the free-flowing solution but upon exposure to the ocular region with the rise in temperature gets converted to a viscoelastic gel. The required temperature for phase transition for a temperature-triggered system is the normal physiological or ocular temperature no external heat for temperature rise is needed 22,23 .

\section{Polymers used in Temperature triggered in-situ systems:}

Chitosan: Chitosan is sugar that is basically produced from the outer skeleton of shellfish, shrimp, lobster, and crabs. Generally used as a dietary supplement. It is made by treating the chitin shells of the above-mentioned crustaceans with an alkaline substance, for example, sodium hydroxide. Chemically it a linear polysaccharide composed of randomly distributed $\beta-(1 \rightarrow 40)$-linked D-glucosamine and N-acetyl-D glucosamine which is deacetylated and acetylated units, respectively. Chitosan possesses advantageous features like mucoadhesiveness, biocompatibility, low cytotoxicity, and 
biodegradability, due to which it is widely used in the biomedical field 23 .

Xyloglucan: Xyloglucan, another polysaccharide, is a hemicellulose which is obtained from the primary cell wall of vascular plants generally from tamarind seeds; therefore, sometimes referred to as tamarind seed polysaccharide. It has a backbone of $\beta \rightarrow 4$-linked glucose residue, most of which is substituted with 1-6 linked xylose side chain. Xyloglucan when it degrades partially by $\beta$-galactosidase forms gel in an aqueous solution 23,24 .

Poloxamers (Pluronic): Poloxamers consist of a non ionic triblock copolymer composed of a central hydrophobic chain of polyoxypropylene (polypropylene oxide) guarded by two hydrophilic chains of polyoxyethylene (polyethylene oxide). Due to the presence of both hydrophilic units(ethylene oxide) and hydrophobic units (propylene oxide), it is amphiphilic in nature. This polymer undergoes a phase transition above $15 \%(\mathrm{w} / \mathrm{w})$ concentration from sol-to-gel at normal body temperature. The most possible reasons for this sol-to-gel transition must be the slow desolvation and increased aggregation of the micelle along with the additional entanglement within the polymer of the polymeric system $^{24,25}$.

\section{EVALUATION}

In-situ gels can be evaluated for the following parameters:

\section{1. pH measurement}

The $\mathrm{pH}$ of the ocular in-situ gel-forming systems is usually measured with the help of a $\mathrm{pH}$ meter which has to be precalibrated. The calibration of the $\mathrm{pH}$ meter is done using buffers of $\mathrm{pH} 7$ and 4 as per the standard procedures ${ }^{26}$.

\section{Gelling capacity}

This evaluation is necessary to know the time taken by the formulation to phase transit from sol to gel. The gelling capacity of the in-situ formulations is usually determined by making use of simulated tear fluid or STF. $0.5-1 \mathrm{ml}$ of a formulation is placed in the $2.0-3.0 \mathrm{ml}$ of STF, and the time taken by the dropped formulation to transform to a gel is noted $^{27}$.

\section{Visual appearance and clarity}

Visual appearance and clarity of ocular in-situ gelling systems are done to check the presence of any small, particulate substances by utilizing fluorescent light against a black and white background ${ }^{28}$.

\section{In-vitro drug release study}

In-vitro drug release study is done by utilizing the Franz diffusion cell. In the receptor compartment, freshly prepared artificial tear fluid is placed. Dialysis membrane is placed in between receptor and donor compartments. The whole assembly is kept on the thermostatically controlled magnetic stirrer to simulate in-vivo conditions and the temperature of medium is maintained at $37^{\circ} \mathrm{C} \pm 0.5^{\circ} \mathrm{C}$. Medium is continuously stirred at $20 \mathrm{rpm}$. $1 \mathrm{ml}$ of a formulation is placed in the donor compartment. $0.5 \mathrm{ml}$ of Sample is withdrawn at a predetermined time interval and after every withdrawal; it is replaced by artificial tear fluid. These Samples are then analysed either on HPLC or Ultraviolet spectrophotometer ${ }^{29}$.

\section{Rheological studies}

Viscosity determination of in-situ formulation is carried out on Brookfield viscometer having a small volume adapter. The measurement of Viscosity is done before and as well as after the gel formation by increasing angular velocity gradually from 0.5 to $100 \mathrm{rpm}^{30}$.

\section{Accelerated stability study}

The stability study for the in-situ formulation is carried out as per ICH guidelines to determine the physical stability of the formulation under accelerated storage conditions. Formulation is subjected to elevated temperatures and humidity conditions of $25 \pm 1^{\circ} \mathrm{C} / 60 \% \mathrm{RH}, 30 \pm 1^{\circ} \mathrm{C} / 65 \% \mathrm{RH}$ and $40 \pm 2 \mathrm{oC} / 75 \pm 5 \% \mathrm{RH}$. Samples are withdrawn at the end of $0,30,60$ and 90 days and then evaluated for active drug content ${ }^{31}$.

\section{Drug content}

For drug content evaluation firstly, in a $100 \mathrm{ml}$ phosphate buffer, 2 to $3 \mathrm{ml}$ of the formulation is dissolved after that the solution is analysed in a UV spectrophotometer ${ }^{31}$.

Table 1: Some of the marketed products of ocular in-situ systems

\begin{tabular}{|l|l|l|l|l|}
\hline Sl. No. & Manufacturing company & Name of the product & Drug used in the formulation & References \\
\hline 1. & $\begin{array}{l}\text { Spectrum } \\
\text { Thea Pharmaceuticals }\end{array}$ & Virgan & Ganciclovir & 32 \\
\hline 2. & Alcon Laboratories Inc. & Pilopine HS & Pilocarpine hydrochloride & 32 \\
\hline 3. & Insite vison & Azasite & Azithromycin & 33 \\
\hline 4. & Merck and Co. Inc & Timoptic-XE & Timolol & 34 \\
\hline
\end{tabular}

\section{SOME OF THE ADVANTAGES OF OCULAR IN- SITU GEL-FORMING SYSTEMS}

- By increasing the precorneal residence time increases the ocular bioavailability

- Provide better fit and housing of the delivery system.

- Due to the decreased loss of the active drug from the ocular region increases accurate dosing.

- Provides prolong, sustained and ultimately controlled drug delivery.
- Due to ease to administer and increased comfort, provides better patient compliance.

- Due to increased precorneal residence time, dosing frequency is significantly decreased.

- Absorption of the drug or trans-barrier (protective barriers of the eye) permeation is enhanced.

- Provides targeted drug delivery in the ocular region and prevents the loss of drug to other ocular tissues ${ }^{35,36}$. 


\section{CONCLUSION}

In ophthalmology, many efforts have been made to develop a delivery system with prolonged residence time in the ocular region, which could ultimately result in the increased ocular bioavailability by making many changes and modifications in the product formulation and product content like by incorporating mucoadhesive polymers, and by modifying the viscosity. One of the major efforts was the development of a delivery system that could transform from sol-to-gel after they are instilled in the ocular region which was named ocular in-situ gel-forming systems. These systems are considered to be the most promising and impressive ones and a large number of studies and experiments are carried out in this field as this system could increase the ocular bioavailability and decrease the systemic toxicity, additionally, offer great patient compliance due to its sustained a prolonged drug-releasing nature resulting in low dosing frequency. However, despite being such an impressive and promising drug delivery system only a hand full of drugs in the form of ocular in-situ gel are in clinically use, having said that, it is important that many other ophthalmic drugs that are crucially needed in ocular patient care and in ophthalmology should be further studied, experimented, and used in the form of an in-situ gel.

\section{REFERENCES}

1. Al-Bazzaz FY, Al-Kotaji MY. Ophthalmic in-situ sustained gel of ciprofloxacin, preparation and evaluation study. Int J App Pharm. 2018; 10(4):153-61. https://doi.org/10.22159/ijap.2018v10i4.26885

2. Patel A, Cholkar K, Agrahari V, Mitra AK. Ocular drug delivery systems: An overview. World journal of pharmacology. 2013; 2(2):47. https://doi.org/10.5497/wjp.v2.i2.47

3. Thimmasetty MK, Mandal S, Prabhushankar GL, Geetha MS. Formulation and evaluation of an in situ gel of amoxicillin trihydrate. Int. J. Pharm. Investig. 2012; 2(2):78-82. https://doi.org/10.4103/2230-973X.100042

4. Kumar SP, Kavitha K, Rupeshkumar M. Recent developments and strategies of ocular in-situ drug delivery systems: a review. Int Pharm Clin Res. 2013; 5:64-71.

5. Rajoria G, Gupta A. In-situ gelling system: a novel approach for ocular drug delivery. AJPTR. 2012; 2:24-53.

6. Ramya DD, Abhirami M, Brindha R, Gomathi S, Vedhahari BN. Insitu gelling system-potential tool for improving therapeutic effects of drugs. International Journal of Pharmacy and Pharmaceutical Sciences. 2013; 5(3):27-30.

7. Agrahari V, Mandal A, Agrahari V, Trinh HM, Joseph M, Ray A, Hadji H, Mitra R, Pal D, Mitra AK. A comprehensive insight on ocular pharmacokinetics. Drug delivery and translational research. 2016 Dec; 6(6):735-54. https://doi.org/10.1007/s13346-016-0339-2

8. NR Galloway. Common Eye Diseases and their Management, Basic Anatomy and Physiology of the Eye.1985. p. 7-15 https://doi.org/10.1007/978-1-4471-3521-0_2

9. Ramaiyan D, \& Vijaya RJ. Ocular Drug Delivery System-A Review. International Journal of Innovative Drug Discovery.2012; 2(1):415.

10. Urtti A. Challenges and obstacles of ocular pharmacokinetics and drug delivery. Advanced drug delivery reviews. 2006 Nov 15; 58(11):1131-5. https://doi.org/10.1016/j.addr.2006.07.027

11. Järvinen $\mathrm{K}$, Järvinen $\mathrm{T}$, Urtti A. Ocular absorption following topical delivery. Advanced drug delivery reviews. 1995 Aug 1; 16(1):3-19. https://doi.org/10.1016/0169-409X(95)00010-5

12. Patel P, Shastri D, Shelat P, Shukla A. Ophthalmic drug delivery system: challenges and approaches. Systematic Reviews in Pharmacy. 2010 Jul 1; 1(2):113. https://doi.org/10.4103/09758453.75042
13. Ripal G, Ananthula HK, Parenky A, Ashim KM.Ocular Drug Delivery. American Association of Pharma ScientistsJ.2012; l(12):348-360. https://doi.org/10.1208/s12248-010-9183-3

14. Varela-Fernández R, Díaz-Tomé V, Luaces-Rodríguez A, CondePenedo A, García-Otero X, Luzardo-Álvarez A, FernándezFerreiro A, Otero-Espinar FJ. Drug delivery to the posterior segment of the eye: Biopharmaceutic and pharmacokinetic considerations. Pharmaceutics. 2020 Mar; 12(3):269. https://doi.org/10.3390/pharmaceutics12030269

15. Jothi M, Harikumar SL, Aggarwal G. In-situ ophthalmic gels for the treatment of eye diseases. International Journal of Pharmaceutical Sciences and Research. 2012 Jul 1; 3(7):1891.

16. Mohanty D, Bakshi V, Simharaju N, Haque MA, Sahoo CK. A review on in situ gel: a novel drug delivery system. Int. J. Pharm. Sci. Rev. Res. 2018; 50(1):175-81.

17. Pandey M, Choudhury H, Bhattamisra SK, Gorain B, Su JS, Tan CL Chin WY, Yip KY. Potential of Stimuli-Responsive In Situ Gel System for Sustained Ocular Drug Delivery: Recent Progress and Contemporary Research. Polymers. 2021 Jan; 13(8):1340. https://doi.org/10.3390/polym13081340

18. Kurniawansyah IS, Rusdiana T, Wahab HA, Subarnas A. In situ opthalmic gel with ion activated system. International Journal of Applied Pharmaceutics. 2019 Jul 7:15-8. https://doi.org/10.22159/ijap.2019v11i4.33072

19. Sriamornsak P. Chemistry of pectin and its pharmaceutical uses: A review. Silpakorn University International Journal. 2003 Jan 1; 3(1-2):206-28.

20. Achouri D, Alhanout K, Piccerelle P, Andrieu V. Recent advances in ocular drug delivery. Drug development and industrial pharmacy. 2013 Nov 1; 39(11):1599-617. https://doi.org/10.3109/03639045.2012.736515

21. Sheshala R, Ming NJ, Kok YY, Singh TR, Dua K. Formulation and characterization of $\mathrm{pH}$ induced in situ gels containing sulfacetamide sodium for ocular drug delivery: A combination of Carbopol®/HPMC polymer. Indian J. Pharm. Educ. 2019 Oct 1; 53:654-2. https://doi.org/10.5530/ijper.53.4.127

22. Laddha UD, Mahajan HS. An insight to ocular in situ gelling systems. Int J Adv Pharm. 2017; 6(2).

23. Wu Y, Liu Y, Li X, Kebebe D, Zhang B, Ren J, Lu J, Li J, Du S, Liu Z. Research progress of in-situ gelling ophthalmic drug delivery system. Asian journal of pharmaceutical sciences. 2019 Jan 1; 14(1):1-5. https://doi.org/10.1016/j.ajps.2018.04.008

24. Padmasri B, nagaraju R, prasanth D. A comprehensive review on in situ gels. International Journal of Applied Pharmaceutics. 2020 Nov 7:24-33. https://doi.org/10.22159/ijap.2020v12i6.38918

25. Nagesh C, Suma N, Sujit P, Chandrasekhara S. Formulation and characterization of thermosensitive in situ gel of moxifloxacin for ocular delivery. Manipal Journal of Pharmaceutical Sciences. 2016 Sep 1; 2(2):4-11.

26. Makwana SB, Patel VA, Parmar SJ. Development and characterization of in-situ gel for ophthalmic formulation containing ciprofloxacin hydrochloride. Results in pharma sciences. 2016 Jan 1; 6:1-6. https://doi.org/10.1016/j.rinphs.2015.06.001

27. Kumar VV, Chetty CM, Reddy YD, Ugandar RE, Gladiola BD. Formulation and in vitro Characterization of Ocular in situ Gels of Valcyclovir. Journal of Pharmaceutical Sciences and Research 2019 Aug $1 ; 11(8): 2974-9$. https://doi.org/10.32553/jbpr.v8i4.641

28. PH AM, Satyananda S. pH-induced in situ gelling system of an anti-infective drug for sustained ocular delivery. Journal of Applied Pharmaceutical Science. 2014 Jan 1; 4(1):11. https://doi.org/10.7324/JAPS.2014.40117

29. Mohan EC, Kandukuri JM, Allenki V. Preparation and evaluation of in-situ-gels for ocular drug delivery. J Pharm Res. 2009 Jun; 2(6):1089-94. 
30. Ramachandra LU, Vikas DG, Gadhave MV, Jadhav SL, Gaikwad DD. Design and development of $\mathrm{pH}$-triggered in situ gelling system of Ciprofloxacin. Int. Res. J Pharmacy. 2012; 3(5):418-22.

31. Gupta C, Juyal V, Nagaich U. Formulation, optimization, and evaluation of in-situ gel of moxifloxacin hydrochloride for ophthalmic drug delivery. International Journal of Applied Pharmaceutics. 2019 Jul 7:147-58. https://doi.org/10.22159/ijap.2019v11i4.30388

32. Majeed A, Khan NA. Ocular in situ gel: An overview. Journal of Drug Delivery and Therapeutics. 2019 Jan 15; 9(1):337-47. https://doi.org/10.22270/jddt.v9i1.2231

33. Soliman KA, Ullah K, Shah A, Jones DS, Singh TR. Poloxamerbased in situ gelling thermoresponsive systems for ocular drug delivery applications. Drug Discovery Today. 2019 Aug 1; 24(8):1575-86. https://doi.org/10.1016/j.drudis.2019.05.036

34. Mammo ZN, Flanagan JG, James DF, Trope GE. Generic versus brand-name North American topical glaucoma drops. Canadian Journal of Ophthalmology. 2012 Feb 1; 47(1):55-61. https://doi.org/10.1016/j.jcjo.2011.12.004

35. Almeida H, Amaral MH, Lobão P, Lobo JM. In situ gelling systems: a strategy to improve the bioavailability of ophthalmic pharmaceutical formulations. Drug discovery today. 2014 Apr 1; 19(4):400-12. https://doi.org/10.1016/j.drudis.2013.10.001

36. Agarwal K. In-situ gel formation for ocular drug delivery system an overview. Asian Journal of Biomedical and Pharmaceutical Sciences. 2011 Oct $1 ; 1(4)$. 